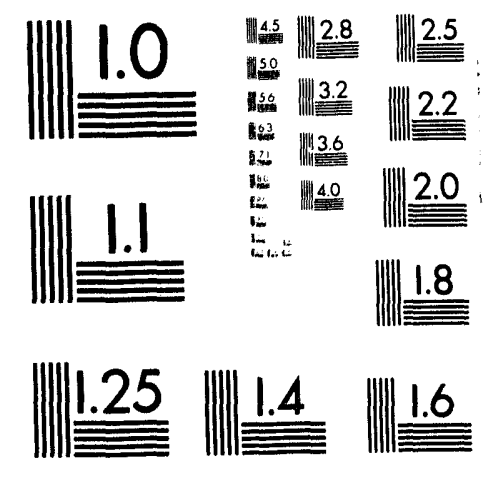




$$
\overrightarrow{\underline{a}}
$$


SIMULATED QUENCHING IN THE GRAND-CANONICAL ENSEMBLE*

\author{
S. R. Phillpot and J. M. Rickman** \\ Materials Science Division \\ Argonne National Laboratory \\ Argonne, Il 60439
}

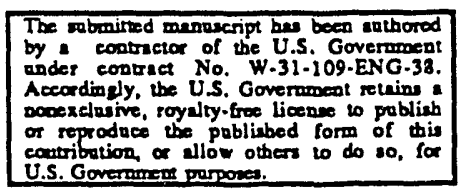

DECEMBER 1991

\begin{abstract}
DISCLAIMER
This report was prepared as an account of work sponsored by an agency of the United States Government. Neither the United States Government nor any agency thereof, nor any of their employees, makes any warranty, express or implied, or assumes any legal liability or responsibility for the accuracy, completeness, or usefulness of any information, apparatus, product, or process disclosed, or represents that its use would not infringe privately owned rights. Reference herein to any specific commercial product, process, or service by trade name, trademark, manufacturer, or otherwise does not necessarily constitute or imply its endorsement, recommendation, or favoring by the United States Government or any agency thereof. The views and opinions of authors expressed herein do not necessarily state or reflect those of the United States Government or any agency thereof.
\end{abstract}

*The effort of SRP was supported by US Department of Energy BES-Materials Science under Contract No. W-31-109-Eng-38. The effort of JMR was supported by the Office of Naval Research under Contract No.N00014-88-F-0019.

**Current address: Department of Materials Science and Engineering, University of Michigan, Ann Arbor, MI 48109. 


\title{
SIMULATED QUENCHING IN THE GRAND-CANONICAL ENSEMBLE
}

\author{
S. R. Phillpot and J. M. Rickman* \\ Materials Science Division \\ Argonne National Laboratory \\ Argonne, IL 60439
}

\begin{abstract}
A formalism for obtaining the zero-temperature structure of mono-component solids in the grand-canonical ensemble is developed. The new methodology, grand-canonical simulated quenching, is validated by simulating systems initially containing a vacancy and an interstitial. As a first application, the reconstruction of a high-angle twist grain boundary in $\mathrm{fcc} \mathrm{Cu}$ is investigated.
\end{abstract}

\section{INTRODUCTION}

One of the purposes of many computer simulations is to calculate the equilibrium structure and properties of a system of atoms that is characterized by a given set of thermodynamic parameters. For example, in order to calculate the stress and energy of an $\mathrm{N}$-atom system at a given volume, $\mathrm{V}$, and temperature, $\mathrm{T}$, one would perform a simulation in the isochoric-isothermal canonical (NVT) ensemble. Similarly, in order to determine the equilibrium number of atoms in a system held at a given volume, temperature and chemical potential, $\mu$, one would perform simulations in the isochoric-isothermal grand-canonical $(\mu \mathrm{VT})$ ensemble.

The variables $N$ and $\mu$ are thermodynamic conjugates as are $V$ and the pressure, $P$. Because simulations at constant volume and at constant pressure are almost equally easy to perform, it would be reasonable to suppose that simulations at constant $\mu$, i.e., in the grand-canonical ensemble (GCE), would not be significantly more difficult to perform than simulations at constant $\mathrm{N}$. This is not the case, however, because whereas $\mathrm{V}$ may change in a continuous way, $\mathrm{N}$ is an integer, i.e., atoms must be added to and removed from a physical system in integer numbers. For a gas or a dilute liquid this presents little problem for simulation because the removal or addition of a single atom has relatively little effect on 
the total energy of the system. Indeed, there have been a number of grand-canonical Monte-Carlo (GCMC) simulations of gases and dilute liquids. ${ }^{1-3}$ As the density of the system increases, however, due w the presence of nearby atoms, the initial energy change on removing or adding a single atom increases and the efficiency of GCMC simulations decreases dramatically. In particular, attempts to insert an atom into dense systems usually fail as there are not many voids into which the atom can go. The maximum density at which GCMC simulations can be efficiently performed has been increased somewhat by using cavity-biased MC. ${ }^{4}$ Nevertheless, for liquids over a wide range of density and for solids, grand-canonical simulations are usually completely impractical.

Recently, to perform simulations in the GCE Cagin and Pettitt 5,6 have proposed a variation of the molecular-dynamics $(\mathrm{MD})$ method, in which the number of atoms is not an integer. In their scheme, at any instant during the simulation there are $\mathrm{N}$ atoms which evolve in the canonical ensemble and an additional fractional atom, the $(\mathrm{N}+1)$ th, which is being added to, or removed from, the system. After this atom has been completely added or removed, another atom insertion or deletion is attempted. They have shown that such a scheme reproduces the statistical mechanics of the grand-canonical ensemble and have validated it with a simulation of a dense liquid. 5,6

While an important breakthrough, there are drawbacks to this scheme. First, the dynamical equations of motion (EOMs) place no bound on the fraction of the $(N+1) t h$ atom. In particular the evolution of the EOMs could result in the fraction of the $(\mathrm{N}+1)$ th atom becoming greater than one or less than zero. Thus, when the fraction of the atom reaches one or zero it becomes necessary to interrupt the dynamical evolution of the system and fix the fraction of the atom to one or zero respectively. Such an interruption of the dynamics of the system corresponds to the application of an external force of unknown magnitude. Second, the $(\mathrm{N}+1)$ th atom is treated in a qualitatively different way from the other $\mathrm{N}$ atoms in the system. It would be more appealing, however, if all atoms were treated equivalently throughout the simulation and were subject to the same dynamics. Moreover, such a scheme involving many simultaneous atom additions and removals might have the practical advantages of both converging in fewer simulation steps and more readily allowing collective effects involving coupled atom insertions and deletions.

In this paper, we present the first steps in a methodology that overcomes the above limitations. In particular, we develop a formalism for determining the zero-temperature structure of mono-component solids in the grand-canonical ensemble. In a canonical- 
ensemble simulation the fundamental object of the simulation is an atom, which has a timedependent position and momentum. Here, the fundamental object of the simulation is a site, which has a time-dependent position and momentum, and to which an atom may be attached. (A site may be considered to be merely a mathematical point in space and is to a certain extent analogous to a Bravais-lattice site.) By choosing a suitable number of sites at the beginning of a simulation, a grand-canonical simulation can be performed with a fixed number of sites but variable number of atoms. In the spirit of Cagin and Pettitt 5,6 , a site is allowed to be empty or occupied by one atom or any fraction in between, thereby allowing the construction of an effective Lagrangian for the position and occupancy of the sites. The equations of motion derived from this Lagrangian can then used in a quenching simulation, which may be considered to be the GCE analog of lattice statics. At each "time" step in the quench, energy is removed from the kinetic degrees of freedom of the system until an energy minimum is reached. We show that each site in the zero-temperature structures obtained using this algorithm is either occupied by exactly one atom or is completely empty. We shall refer to the scheme that generates these zero-temperature structures as grand-canonical simulated quenching (GCSQ).

The rest of this paper is organized as follows. In the next Section we develop a Lagrangian for simulations of solids in the grand-canonical ensemble. In Section III we derive equations of motion from this Lagrangian and investigate their zero-temperature solutions. The methodology is validated in Section IV by using GCSQ to obtain the zerotemperature structure of systems initially containing point defects. We then investigate, in Section $\mathrm{V}$, the structure of a high-angle twist grain boundary on the (110) plane of $\mathrm{Cu}$ (as represented by a Lennard-Jones potential) using GCSQ. Section VI contains a brief discussion and an outlook.

\section{FORMALISM FOR SIMULATIONS IN THE GRAND-CANONICAL ENSEMBLE}

The thermodynamic description of a system subject to a fixed $\mu, \mathrm{V}$, and $\mathrm{T}$ is given by the grand-canonical ensemble. ${ }^{7}$ Just as the internal energy, E, is the natural heat function in the NVT ensemble, and the enthalpy, $\mathrm{H}=\mathrm{E}+\mathrm{PV}$, is the natural heat function in the NPT ensemble, the natural heat function, $L$, in the grand-canonical $(\mu V T)$ ensemble is

$\mathrm{L}=\mathrm{E}-\mu \mathrm{N}$ 
Because it was first introduced by $\mathrm{Hill}^{7}, \mathrm{~L}$ is known as the Hill energy.

In this paper, we confine our attention to mono-component systems, for which the chemical potential is equal to the Gibbs free energy per atom which, at zero temperature and pressure, is just the internal energy per particle. Thus, for a zero-temperature perfect crystal $\mathrm{L}$ vanishes. By contrast, for an inhomogeneous system at zero temperature $\mathrm{L}>0$.

Let us write down the Hill energy for a system of atoms in the ( $\mu \mathrm{VT})$ ensemble interacting via a pair potential, $U\left(r_{i j}\right)$, with $r_{i j}=\left|r_{i}-r_{j}\right|$. (While here we consider a pair potential for notational simplicity, the argument is quite general.) Equation 2.1 then becomes

$L=\frac{1}{2} \sum_{i=1}^{N} \sum_{\substack{j=1 \\ j \neq i}}^{N} U\left(r_{i j}\right)-\mu N$

In order to perform simulations in the grand-canonical ensemble, in principle all one now has to do is to allow atom additions and deletions. However, as discussed in the Introduction, while such simulations are practical for gases and dilute liquids, they are impractical for dense fluids and solids.

To develop a formalism for the simulation of solids in the GCE, we make two departures from canonical-ensemble simulation schemes for systems interacting via continuous potentials.

(i) We consider a solid of mobile atoms as being formed from $\mathrm{M}$ mobile sites, which may or may not be occupied. These sites may be considered to be merely mathematical points in space, to which atoms can be attached. Thus, rather than describing a solid as an $\mathrm{N}$-atom system with atom $i$ described by the six position and momentum coordinates $\left(r_{i}, p_{i}\right)$, the solid will be described as a system of $M$ sites $(M>N)$, the ith site being described by its position, $r_{i}$, its momentum $p_{i}$ and by the number of atoms at the site (the 'occupancy' of the site).

(ii) The occupancy of the each site is taken to be a time-dependent non-integer quantity; the occupancy of the ith site is $x_{j}$ where $0 \leq x_{i} \leq 1$. 
By choosing an appropriate number of sites at the beginning of the simulation (i) will allow us to perform simulations witi a fixed number of sites but a variable number of atoms. By allowing the occupancy of any site to be a fractional variable, changes in occupancy may be made continuously with resultantly small incremental changes in the energy of the system. Moreover, since it is a continuous variable, equations of motion for $x_{i}$ may be derived. The price that must be paid for this is, of course, that the occupancy of any site is not necessarily an integer at all simulation steps. However, as will be shown below, the above assumptions lead to zero-temperature structures in which there are no fractionally occupied sites: at the end of a converged simulation, each site is either occupied by exactly one atom or is empty.

The number of atoms in the solid, $\mathrm{N}$, which is less than or equal to the number of sites in the system, $M$, is given by:

$N=\sum_{i=1}^{M} x_{i}$

Here and in the following, the time variation of most of time-dependent quantities is not explicitly displayed. The grand-canonical heat function, L, by analogy with Eq. 2.2 is

$L=\frac{1}{2} \sum_{i=1}^{M} \sum_{\substack{j=1 \\ j \neq 1}}^{M} x_{i} x_{j} U\left(r_{i j}\right)-\mu \sum_{i=1}^{M} x_{i}$

While it is possible to perform Monte-Carlo simulations using the heat function in Eq. 2.4, here we shall construct a Hamiltonian incorporating the potential energy function in Eq. 2.4 and appropriate kinetic energy terms, thereby allowing us to generate equations of motion for the positions, momenta and occupancies of the sites. Such dynamical simulation are more convenient for this initial study because they allow us to easily test the correctness of the algorithm by monitoring "energy" conservation.

First, however, we recall that in postulating fractional occupancy of sites, the constraint $0 \leq x_{i} \leq 1 \forall$ was assumed. In general, it is not easy to handle such non- 
holonomic constraints. However, in this case the constraint is easily imposed by simply making a change of variable from $x$ to $\theta$, where

$$
\mathrm{x}_{\mathrm{i}}=\frac{1}{2}\left(\cos \theta_{\mathrm{i}}+1\right)
$$

Here, although $\theta_{i}$ is unbounded, Eq. 2.5 incorporates the constraint $0 \leq x_{i} \leq 1$. The choice of change of variables in Eq. 2.5 is not unique but is motivated by close analogies, outlined in the Appendix, between this model and the lattice-gas, Ising-spin and Heisenberg-spin models.

A kinetic energy can be assigned to the sites, here all assumed to have mass $m$, and is given by:

$\mathrm{T}_{\mathrm{T}}=\frac{1}{2} \mathrm{~m} \sum_{\mathrm{i}=1}^{\mathrm{M}} \dot{\mathbf{r}}_{\mathrm{i}}^{2}$

To generate dynamics for the occupancy of the sites we write a 'kinetic energy' function:

$\mathrm{T}_{\theta}=\frac{1}{2} \mathrm{Q} \sum_{\mathrm{i}=1}^{\mathrm{M}} \dot{\theta}_{\mathrm{i}}^{2}$

where $Q$ is a fictional 'mass' parameter, with dimensions such that $T_{\theta}$ has units of energy. The magnitude of $Q$ may be chosen so as to optimize the convergence of the simulation.

The total energy function for the system of $M$ sites is, then,

$$
\begin{array}{r}
E=\frac{1}{2} Q \sum_{i=1}^{M} \dot{\theta}_{i}^{2}+\frac{1}{2} m \sum_{i=1}^{M} \dot{r}_{i}^{2}+\frac{1}{8} \sum_{i=1}^{M} \sum_{\substack{j=1 \\
j \neq i}}^{M}\left(\cos \theta_{i}+1\right)\left(\cos \theta_{j}+1\right) U\left(r_{i j}\right) \\
-\frac{1}{2} \mu \sum_{i=1}^{M}\left(\cos \theta_{i}+1\right),
\end{array}
$$

and the associated Hamiltonian is given by 


$$
\begin{gathered}
H=\frac{1}{2 Q} \sum_{i=1}^{M} \pi_{i}^{2}+\frac{1}{2 m} \sum_{i=1}^{M} p_{i}^{2}+\frac{1}{8} \sum_{i=1}^{M} \sum_{\substack{j=1 \\
j \neq i}}^{M}\left(\cos \theta_{i}+1\right)\left(\cos \theta_{j}+1\right) U\left(r_{i j}\right) \\
-\frac{1}{2} \mu \sum_{i=1}^{M}\left(\cos \theta_{i}+1\right)
\end{gathered}
$$

where $\pi_{\mathrm{i}}$ is the conjugate momentum to $\theta_{\mathrm{i}}$ and $\mathrm{p}_{\mathrm{j}}$ is the conjugate momentum to $\mathbf{r}_{\mathrm{i}}$. Since this Hamiltonian is not explicitly time dependent, $\mathrm{H}$ is a conserved quantity.

The Lagrangian, $\mathcal{L}$, may be derived fror 1 the above Hamiltonian in the usual way:

$$
\begin{aligned}
& C=\frac{1}{2} Q \sum_{i=1}^{M} \dot{\theta}_{i}^{2}+\frac{1}{2} m \sum_{i=1}^{M} \sum_{\alpha=1}^{3} \dot{r}_{i \alpha}^{2}-\frac{1}{8} \sum_{i=1}^{M} \sum_{\substack{j=1 \\
j \neq i}}^{M}\left(\cos \theta_{i}+1\right)\left(\cos \theta_{j}+1\right) U\left(r_{i j}\right) \\
& +\frac{1}{2} \mu \sum_{i=1}^{M}\left(\cos \theta_{i}+1\right) \text {. }
\end{aligned}
$$

In Eq. 2.10 the Cartesian components, $r_{i \alpha}$, of the vectors $r_{i}$ are explicitly shown.

III

\section{EQUATIONS OF MOTION AND THEIR ZERO-TEMPERATURE SOLUTION}

The Euler-Lagrange equations may be used to derive the equations of motion for $\theta_{i}$ and $r_{i}$ from Eq. 2.10. They are:

$$
\begin{aligned}
& Q \ddot{\theta}_{i}=\frac{1}{4} \sin \theta_{i} \sum_{\substack{j=1 \\
j \neq i}}^{M}\left(\cos \theta_{j}+1\right) U\left(r_{i j}\right)-\frac{\mu}{2} \sin \theta_{i} \\
& m \ddot{r}_{i \alpha}=-\frac{1}{8} \sum_{\substack{j=1 \\
j \neq i}}^{M}\left(\cos \theta_{i}+1\right)\left(\cos \theta_{j}+1\right) \frac{\partial U\left(r_{i j}\right)}{\partial r_{i} \alpha}
\end{aligned}
$$

Conventional canonical-ensemble molecular dynamics may be recovered from Eqs. 3.1a and $3.1 b$ by setting $\cos \theta_{i}=1\left(\sin \theta_{i}=0\right)$, and hence $x_{i}=1$ for all $i$. 
Consider a calculation of the zero-temperature equilibrium structure of a system whose evolution is described by Eqs. 3.1a and 3.1b. To do this we must find stationary solutions with $\ddot{\mathrm{r}}_{\mathrm{i}}=0$ and $\ddot{\theta}_{\mathrm{i}}=0$. There are two stationary solutions to Eq. 3.1a. They are:

$\sin \theta_{\mathrm{i}}=0$

and

$\mu=\frac{1}{2} \sum_{j=1}^{M}\left(\cos \theta_{j}+1\right) U\left(r_{i j}\right), \quad \sin \theta_{i} \neq 0$

The first solution (Eq. 3.2) leads to two different physical situations. For $\cos \theta_{\mathrm{i}}=$ +1 the ith site is occupied $\left(x_{i}=1\right)$. For $\cos \theta_{i}=-1$ the ith site is unoccupied $\left(x_{i}=0\right)$. Notice that these solutions are independent of $j$, i.e., the system may contain a mixture of occupied and unoccupied sites. If all of the sites are unoccupied then there are no atoms in the system. Trivially, this is an energy minimum with $L=0$. There is a second solution with $L=0$, which corresponds to a perfect crystal. Depending on the initial choice of the number of sites this will have only some, or all of the sites occupied.

Analysis of the second stationary solution is only a little more complicated. Recall that the zero-temperature chemical potential is simply the zero-temperature perfect-crystal cohesive energy per atom, $U_{0}$. However, the right-hand side of Eq. 3.3 is the energy of atom i. Thus, the only solution to Eq. 3.3 is if jth site is occupied by exactly one atom or is empty such that all the occupied sited form a perfect-crystal lattice.

The stationary solution of Eq. $3.1 \mathrm{~b}$ must simultaneously be obtained. For $x_{i}=0$ or $x_{j}=0$ Eq. $3.1 b$ vanishes. For $x_{i}=1$ and $x_{j}=1$, Eq. $3.1 b$ yields:

$$
\sum_{\substack{j=1 \\ j \neq i \\ \text { occ. }}}^{M} \frac{\partial U\left(r_{i j}\right)}{\partial r_{i \alpha}}=0
$$

i.e., the net force on each occupied site due to the other occupied sites is zero, which is the usual equilibrium constraint on atom positions. 
In summary, the above analysis shows that the zero-temperature stationary solution to the equations of motion consists of $\mathrm{N}$ of the $\mathrm{M}$ sites being occupied by exactly one atom, the remaining M-N sites being completely empty, and the force on each occupied site due to its occupied neighbors being zero. Importantly, in the stationary state, there are no sites occupied by only a fraction of an atom.

In Sec IV we shall validate the above methodology by performing grand-canonical simulated quenches on a perfect crystal and a perfect crystal containing a single vacancy and a single interstitial.

\section{VALIDATION OF THE GCSQ METHOD: THE PERFECT CRYSTAL AND THE CRYSTAL WITH POINT DEFECTS}

In all of our simulations the atoms interact via a Lennard-Jones potential parameterized to copper $(\varepsilon=0.167 \mathrm{eV}, \sigma=2.315 \AA)$, shifted smoothly to zero and cut off 8,9

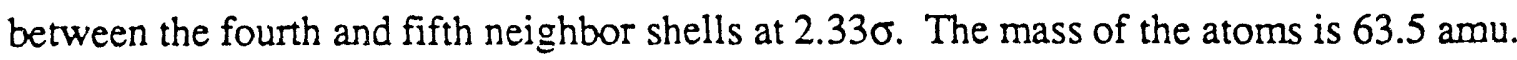
For this potential and these parameters the zero-temperature lattice constant, a, has the value $a=3.616 \AA$, and the cohesive energy per atom, $U_{0}$, has the value $U_{0}=-1.0378 \mathrm{eV}$. Recall that the zero-temperature chemical potential is equal to the cohesive energy, i.e., $\mu=-$ $1.0378 \mathrm{eV}$. The melting temperature for this potential is approximately $1200 \mathrm{~K}$. The fictional mass, $Q$, associated with the occupancy degrees of freedom was chosen to be 262 amu $\AA^{2}$. The effects of changing this this rather arbitrary value of $Q$ were not investigated, but should only effect the rate at which the simulation converges. In all the simulations the 'time' step was sufficiently small that in trial simulations in the $\mu V E$ ensemble, the Hill energy for a system evolving with three-dimensionally periodic border conditions was constant to six significant figures over simulations of a few thousand time steps. (In none of our simulations do the dynamics correspond to any real-time evolution of the system but are merely a technique for determining the zero-temperature structure.) In each of the simulations described below, the grand-canonical simulated quench to zero temperature was performed by removing a small fraction $(0.125 \%)$ of the kinetic energy in the realspace and occupancy-space degrees of freedom at every time step. As an alternative method of energy removal one could add a phenomenological damping force to the equations of motion (Eqs. 3.1a and 3.1b). However, because we are only interested in the final zero-temperature equilibrium state the exact method of removing energy from the system is unimportant. 


\section{IV.1 THE PERFECT CRYSTAL}

The most basic test of the GCSQ scheme is to investigate the stability of the zerotemperature perfect crystal. As an initial condition, we considered 108 sites arranged as an fcc lattice, such that if each site were occupied by exactly one atom, a zero-temperature perfect crystal would be formed. Each site was initially assigned a random real-space velocity, such that the initial temperature of the sites was $T_{i n i t}$. Each site was also assigned an initial occupancy of less than unity, chosen randomly from a sample with a mean of $\mathbf{x}_{\text {init }}$ and a standard deviation of $\sigma_{\text {init }} \approx 0.05$. For the range of initial temperatures investigated, $0 \mathrm{~K}<\mathrm{T}_{\text {init }}<300 \mathrm{~K}$, and for $\mathrm{x}_{\text {init }} \leq 0.5$, after a quench of 5000 time steps the total number of atoms on the sites was found to be zero: each site was empty and $L=0$. For the same range of initial temperatures, but for $x_{\text {init }} \geq 0.5$, a similar quench produced a perfect crystal of 108 occupied sites, again with $L=0$. These results are entirely consistent with the analytic results of the previous section.

\section{IV.2 THE OCTAHEDRAL INTERSTITIAL}

A structure containing an octahedral interstitial was formed by adding to a perfect crystal of 108 atoms an atom at the center of one of the cubic conventional unit cells. On quenching with constant number of atoms (109), the system relaxed to form the dumbbell, or [100] split, structure. ${ }^{10}$

This relaxed structure was used as an input into the GCSQ scheme. The total number of sites in the system was 109, each initially at the position of one of the atoms in the relaxed structure. Initially each site had an occupancy a little less than one; thus the initial total number of atoms was somewhat less than 109. Figure 1 shows the number of atoms in the system and the Hill energy as a function of the number of simulation steps. We see that the final state has exactly 108 atoms and $L=0$; i.e., it is a perfect crystal.

Notice that during the simulation the total number of atoms on the sites decreases below 108. This is a kinetic effect and presumably more easily allows the expulsion of the interstitial atom. An analysis of the trajectories of the system obtained in several different simulation runs shows that the final 108-atom equilibrium structure can be obtained by different paths. In some cases the atom initially at the octahedral site is expelled during the 
course of the simulation while, in others, a neighboring atom is expelled and the interstitial atom moves into the void left by it.

\section{IV.3 THE MONOVACANCY}

A 107-atom system consisting of a perfect crystal with a single vacancy was relaxed at zero temperature in the canonical ensemble. As a simple test of the stability of a monovacancy, GCSQ was performed on this relaxed system with 108 sites. 107 of these sites were placed at the relaxed atum positions and given occupancies close to 1; the 108th site was placed at the position of the vacancy and given an occupancy close to 0 . As expected, GCSQ resulted in the occupancy of the vacant site increasing to one, thereby forming a perfect crystal of 108 atoms.

Of course, this is a rather easy test to pass as the system is biased by placing an atom at the vacant site. In a more general inhomogeneous solid (for example, one containing a grain boundary, dislocation or a free surface) one would not know where the addition or removal of an atom might lower the energy of the system. To consider such a complicated system, it is necessary to have an algorithmic way of choosing the number and location of additional sites. The Voronoi construction provides such a procedure. ${ }^{9}$ The Voronoi (or Wigner-Seitz) construction for an $\mathrm{N}$ atom system consists of generating $\mathrm{N}$ space-filling cells, all points in the interior of a given cell being closer to the atom at the center of the cell than to any other atom. The edges of the cells are thus lines equidistant from two atoms; the vertices of the cells are points equidistant from three or more atoms. Thus, the vertices of the Voronoi cells are the centers of the largest unoccupied regions in the system and make a natural choice for the location of additional sites. Of course, in the equilibrated system most, if not all, of these sites will be unoccupied.

For a perfect fcc crystal there are three times as many distinct Voronoi vertices as there are atoms; thus, for a perfect crystal of 108 atoms there are 324 vertices. Each vertex is at an octahedral or a tetrahedral interstitial site. For a 108-atom system with a monovacancy (i.e. a total of 107 atoms) there are 317 distinct Voronoi vertices. We performed a simulation for a system consisting of $107+317=424$ sites. Each of the 107 sites from the relaxed monovacancy structure was assigned $x_{i n i t}=0.90 \pm 0.05$. Each of the 317 sites generated from the Voronoi construction was assigned $x_{\text {inil }}=0.0001$. After GCSQ, 316 of the 317 sites generated from the Voronoi construction had occupancy zero; 
the other site had an occupancy of one. Thus the final number of atoms in the 324 site system was 108 and the system had $\mathrm{L}=0$ : i.e., a perfect crystal was formed.

\section{ZERO-TEMPERATURE STRUCTURE OF A HIGH-ANGLE GRAIN BOUNDARY}

To illustrate the utility of this new methodology for investigating the zerotemperature structure of a structurally more complex inhomogeneous system we consider a high-angle twist grain boundary (GB) on a low-index plane of fcc $\mathrm{Cu}$. As an initial model geometry we choose a point-defect free GB derived from the coincident-site-lattice (CSL). The (110) $\theta=31.59^{\circ}$ ( 227$) \mathrm{GB}$ studied here is obtained by rotating one perfect semi-crystal relative to another by an angle of $31.59^{\circ}$ about the (110) plane normal. It has a rectangular planar repeat unit with an area which is $\Sigma=27$ that of the corresponding primitive planar unit cell $(\Sigma=1)$ on the (110) plane in a perfect single crystal. The system is oriented with the planar normals along the $z$-axis and, consequently, the $x$-y plane is parallel to the $G B$ plane. Because of the presence of the interface, there is no periodicity in the z-direction. However, in the $x-y-$ plane, the structure is periodic.

The zero-temperature structure and energy of this grain boundary have previously been determined in the canonical ensemble by iterative energy minimization ("lattice statics") using a Region I-Region II scheme to simulate an isolated grain boundary. In this scheme, the computational cell is periodic in the $x-y$ plane, but the GB region is embedded in the $z$-direction between two rigid block of atoms. ${ }^{11-13}$ The simulation was performed under zero-stress in the $z$ direction. A substantial volume expansion at the grain boundary was observed and the grain boundary energy was found to be $1052 \mathrm{~mJ} \mathrm{~m}^{-2}$. ${ }^{14}$ For future reference, this GB structure will be denoted the V-structure (for volume-relaxed structure).

A similar Region I -Region II scheme was used for the GCSQ. As an initial structure we chose the unrelaxed grain boundary with no volume expansion. Since this boundary has a higher atomic density than that of the V-structure, it is expected that atoms will be removed from, rather than added to, the system when the numbcr of atoms is allowed to vary. Therefore, the initial sites were arranged to be coincident with the locations of the unrelaxed atoms. No additional sites were added. Further, because we expect any reconstruction to take place very close to the grain boundary, initially all sites except those in the two planes adjacent to the GB were given an occupancy close to unity. 
Atoms in the two pianes adjacent to the interface were assigned occupancies with a mean of $x_{\text {init }}$ and a standard deviation of $\sigma_{\text {init }} \approx 0.05$. The lowest-energy configuration (designated here as the $\mathrm{N}$-structure), obtained for $\mathrm{x}_{\text {init }}=0.63$, had energy $1019 \mathrm{~mJ} \mathrm{~m}^{-2}$ which is $33 \mathrm{~mJ}$ $\mathrm{m}^{-2}$ less than the of the $\mathrm{V}$-structure. The 'temporal' evolution of the total number of atoms in the system and the Hill energy during the course of this simulation are shown in Fig. 2. Of the $54(2 \times 27)$ atoms in the two planes adjacent to the grain boundary, 16 were removed during the GCSQ. Lattice-statics simulations at zero stress in the $\mathrm{z}$ direction for this $\mathrm{N}$-structure resulted in a small volume contraction and a further reduction of the $\mathrm{GB}$ energy to $992 \mathrm{~mJ} \mathrm{~m}^{-2}$ (the NV-structure) which is $60 \mathrm{~mJ} \mathrm{~m}^{-2}$ (or 6 percent) less than that of the V-structure.

GCSQ was also performed in which the occupancy of the sites in the two planes of atoms either side of the volume-unrelaxed GB structure were set to $x_{i n i t}<1$. In other simulations, extra sites were added at each of the vertices of the Voronoi cells near the GB. In no case, for a variety of values of $x_{\text {init }}$, was a configuration obtained with energy lower than that of the system described above.

We now briefly compare the V-structure with the NV-structure. A measure of the density of a system containing a grain boundary is the volume excess per unit area, $\delta \mathrm{V} / \mathrm{A}$, of a system containing an interface over a perfect crystal with an equal number of atoms. For the $\mathrm{V}$-structure $\delta \mathrm{V} / \mathrm{A}=0.179 \mathrm{a},{ }^{14}$ for the $\mathrm{NV}$-structure $\delta \mathrm{V} / \mathrm{A}=0.149 \mathrm{a}$; i.e., the NVstructure is substantially more dense in the interface region than the $\mathrm{V}$-structure. That there are also significant qualitative differences in the $\mathrm{NV}$ - and $\mathrm{V}$-structures at the atomic level is illustrated in Fig. 3, which shows edge-on views of the two grain boundaries. For clarity, each individual atomic plane is denoted by a different symbol. In this projection, the $\mathrm{V}$ structure is symmetric about $z=0$ (the GB-plane) but has no atoms at the GB plane. It is clear that the NV-structure is qualitatively different from the V-structure in that there is a plane of atoms (denoted by crosses) at the GB-plane. The small asymmetry in this structure arises from the dynamics $c$ the simulation: the absolute (lower) energy minimum is probably symmetric.

\section{DISCUSSION AND OUTLOOK}

In this paper we have developed the method of grand-canonical simulated quenching. The methodology was validated at zero temperature by considering simple point-defected systems. Its general uility for calculating the structure of inhomogeneous 
systems was illustrated by considering the reconstruction of a point-defect free high-angle twist grain boundary.

It remains an important open question as to whether the Lagrangian derived in Sec. II and its associated equations of motion (Eqs. 3.1) reproduce the grand-canonical ensemble at non-zero temperatures. The answer to that question does not, however, affect the validity of GCSQ for the investigation of zero-temperature properties. At zero temperature, the only requirement for a valid scheme is that the number and positions of atoms in a solid be allowed to change such that the grand-canonical heat function is minimized. The scheme described in this paper fulfills this criterion.

GCSQ need not be significantly more memory or CPU intensive than canonicalensemble molecular-dynamics (MD) simulations. In $\mathrm{MD}$ the system is characterized by the $6 \mathrm{~N}$ atomic positions and momenta. In GCSQ the system is characterized by the $8 \mathrm{M}$ degrees of freedom, $\mathrm{OM}$ of which are associated with the positions and momenta of the sites and $2 \mathrm{M}$ associated with the site occupancies and their conjugate momenta. Thus, rather than having to integrate $3 \mathrm{~N}$ equations, it is now necessary to integrate $4 \mathrm{M}$ equations of motion. However, since. in most simulations $M$ need not be much larger than $N$ the computational penalty for allowing site $\propto$ ccupancies to change can be quite small. Moreover, as can be seen from Figures 1 and 2 runs of less than 10,000 steps are sufficient to result in equilibration. This is to be compared with grand-canonical Monte-Carlo simulations which, for even moderately dense liquids, very long runs are required because most of the attempted atom insertions and deletions are not accepted. ${ }^{15}$ Also the simulations of liquids by Cagin and Pettitt required runs of hundreds of thousands of steps. 5,6

In the grain-boundary study we noted that the final state depends quite strongly on the initial conditions. This, of course, arises because during a quench the system can easily become trapped in a metastable minimum. Recently, Press and Teukolsky have developed a methodology for performing simulated annealing with continuous (rather than discrete) variables. ${ }^{16}$ We anticipate that simulated annealing of this type might resolve this problem and allow the absolute energy minimum to be reached more easily.

Finally, a further important extension of the GCSQ method will be to multicomponent systems, which will enable the investigation of questions in areas of interfacial 
chemistry such as dissimilar materials interfaces, epitaxy and segregation to grainboundaries.

\section{ACKNOWLEDGEMENTS}

We are happy to acknowledge many useful conversations with J. Eggebrecht, J. A. Jaszczak, R. Kulver and D. Wolf. We thank N. Trivedi and J. Lee for a useful discussion about spin models. This work was supported in part through a grant of computer time from the National Energy Research Supercomputer Center. The effort of SRP was supported by the US Department of Energy BES-Materials Science under Contract No.W-31-109-Eng38 and the effort of JMR was supported by the Office of Naval Research under Contract No.N00014-88-F-0019. 


\section{APPENDIX A: RELATION TO LATTICE GAS AND SPIN MODELS}

In this brief Appenai: we highlight some of the similarities between the model discused here and more familiar lattice-gas and spin models.

To see the analogy between Eq. 2.4 and the lattice-gas (LG) model consider the case in which $U\left(r_{j}\right)$ is a nearest-neighbor potential. If the positions of the sites are periodic in space and fixed in time, then:

$$
\begin{aligned}
U\left(r_{i j}\right)=U\left(r_{i j}^{0}\right) & =U_{0}(j \text { a nearest neighbor of } \mathrm{i}) \\
& =0 \quad \text { (otherwise) }
\end{aligned}
$$

If the occupancy of any site is required to be an integer, $l_{i}$, which can take the values 0 and 1 only then Eq. 2.4 may be rewritten as:

$L=\frac{1}{2} U_{0} \sum_{i=1}^{M} \sum_{j}^{\prime} l_{i} l_{j}-\mu \sum_{i=1}^{M} l_{i}$

where $\Sigma^{\prime}$ indicates a restricted sum over the nearest neighbors of i. Equation A. 2 is the lattice gas model 7 .

As is well-known, ${ }^{7}$ the LG model may be transformed to the equivalent magnetic problem by making the transformation:

$\mathrm{l}_{\mathrm{i}}=\frac{1}{2}+\mathrm{S}_{\mathrm{i}}^{(\mathrm{z})}$

where $S_{i}^{(z)}$ is the $z$ component of the spin on the ith site and make take the values of $\pm \frac{1}{2}$.

For the magnetic problem the Hamiltonian may be written as

$L=\frac{1}{2} U_{0} \sum_{i=1}^{M} \sum_{\substack{j=1 \\ j \neq i}}^{M}\left(S_{i}^{(z)}+\frac{1}{2}\right)\left(S_{j}^{(z)}+\frac{1}{2}\right)-\mu \sum_{i=1}^{M}\left(S_{i}^{(z)}+\frac{1}{2}\right)$ 
This may be rearranged (and variables changed) to obtain the Ising model:

$L=\frac{1}{2} J \sum_{i=1}^{M} \sum_{\substack{j=1 \\ j \neq i}}^{M} S_{i}^{(z)} S_{j}^{(z)}-H \sum_{i=1}^{M} S_{i}^{(z)}-E_{0}$

where $\mathrm{J}=\mathrm{U}_{0}, \mathrm{H}=\frac{\mathrm{UZ}}{2}-\mu$ and $\mathrm{E}_{0}=\frac{1}{2} \mathrm{~N}_{0}\left(\frac{\mathrm{UZ}}{4}-\mu\right)$

The reason for the change of variables in Eq. 2.5 is now clear: a Heisenberg-type spin model can be produced from an Ising-type model by making $\mathrm{S}_{\mathrm{i}}^{(\mathrm{z})}$, into a continuous variable by defining:

$\mathrm{S}_{\mathrm{i}}^{(\mathrm{z})}=\frac{1}{2} \cos \theta_{\mathrm{i}}$

i.e.,

$\mathrm{l}_{\mathrm{i}}=\frac{1}{2}\left(\cos \theta_{\mathrm{i}}+1\right)$

which is Eq. 2.5. 


\section{REFERENCES}

*Current address: Department of Materials Science and Engineering, University of Michigan, Ann Arbor, MI 48109.

1. G. E. Norman and V. S. Filinov, High Temp (USSR) 7, 216 (1969).

2. L. A. Rowley, D. Nicholson and N. G. Parsonage, J. comput. Phys. 26, 66 (1978).

3. J. Yao, R. A. Greenkorn and K. C. Chao, Mol. Phys. 46, 587 (1982).

4. M. Mezei, Molec. Phys. 40, 901 (1980).

5. T. Cagin and B. M. Pettitt, Mol. Phys. 72, 169 (1991).

6. T. Cagin and B. M. Pettitt, Molec. Simul. 6, 5 (1991).

7. T. L. Hill, Statistical Mechanics (McGraw-Hill, New York, 1956).

8. S. D. Stoddard and J. Ford, Phys. Rev. A 8, 1504 (1973).

9. M. P. Allen and D. J. Tildesley, Computer Simulation of Liquids (Clarendon Press, Oxford, 1987).

10. C. P. Fynn, Point Defects and Diffusion (Clarendon Press, Oxford, 1972).

11. D. Wolf, Acta Metall. 32, 245 (1984).

12. D. Wolf, Acta Metall. 32, 735 (1984).

13. D. Wolf, J. Am. Cer. Soc. 67, 1 (1984).

14. D. Wolf, Acta. Metall. 37, 2823 (1989).

15. D. Nicholson, CCP5 Quarterly 11, 19 (1984).

16. W. H. Press and S. A. Teukolsky, Computers in Physics 5, 426 (1991). 


\section{FIGURE CAPTIONS}

Fig.1

Fig. 2

Fig. 3
Number of atoms $(N)$ and Hill energy per site (L/M) as a function of the number of 'time' steps for a 109 site system initially containing an octahedral interstitial. Notice that in the course of the simulation the number of atoms dips below the 108 atoms in the final perfect -crystal configuration. The Hill energy rapidly converges to the perfect crystal value of $L=0$.

Number of atoms $(N)$ and Hill energy per site (L/M) as a function of the number of time steps for a 648 site system initially containing a (110) $\theta=31.59^{\circ}(\Sigma 27)$ grain boundary. The non-zero final value of the Hill energy of this $\mathrm{N}$-structure corresponds to a grain boundary energy of $1019 \mathrm{~mJ} \mathrm{~m}^{-2}$.

Edge-on view of two relaxed structures of the (110) $\theta=31.59^{\circ}$

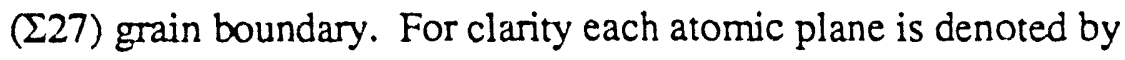
a different symbol.

(i) Grain boundary relaxed in the canonical ensemble under zero stress in the z-direction (the V-structure). The energy of this structure is $1052 \mathrm{~mJ} \mathrm{~m}^{-2}$.

(ii) Grain boundary structure generated using GCSQ and then relaxed in the canonical ensemble under constant stress in the $z$ direction (the NV-structure). The energy of this structure is 992 $\mathrm{mJ} \mathrm{m} \mathrm{m}^{-2}$. 

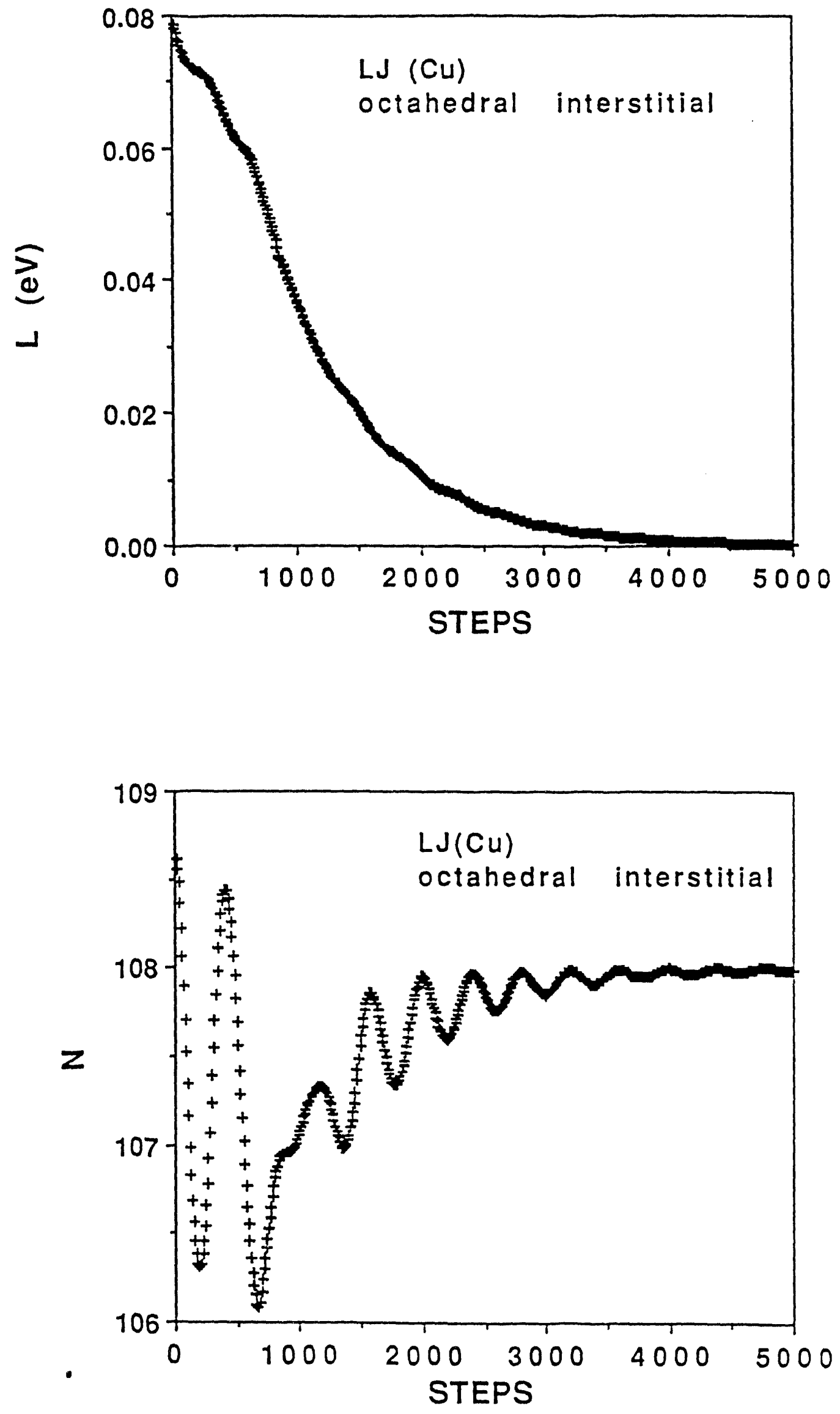

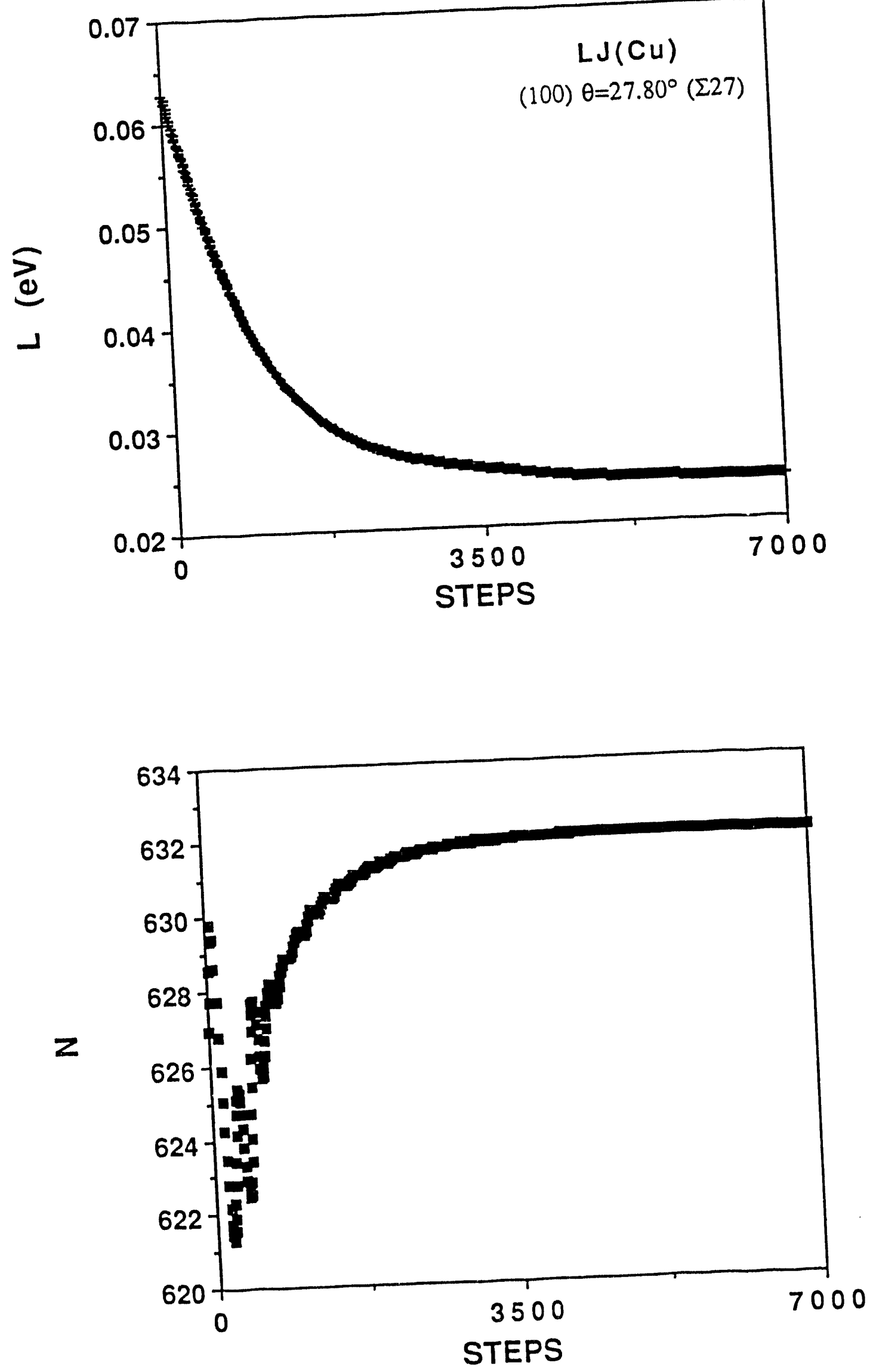

$F_{15} .2$ 
(a)

V-structure
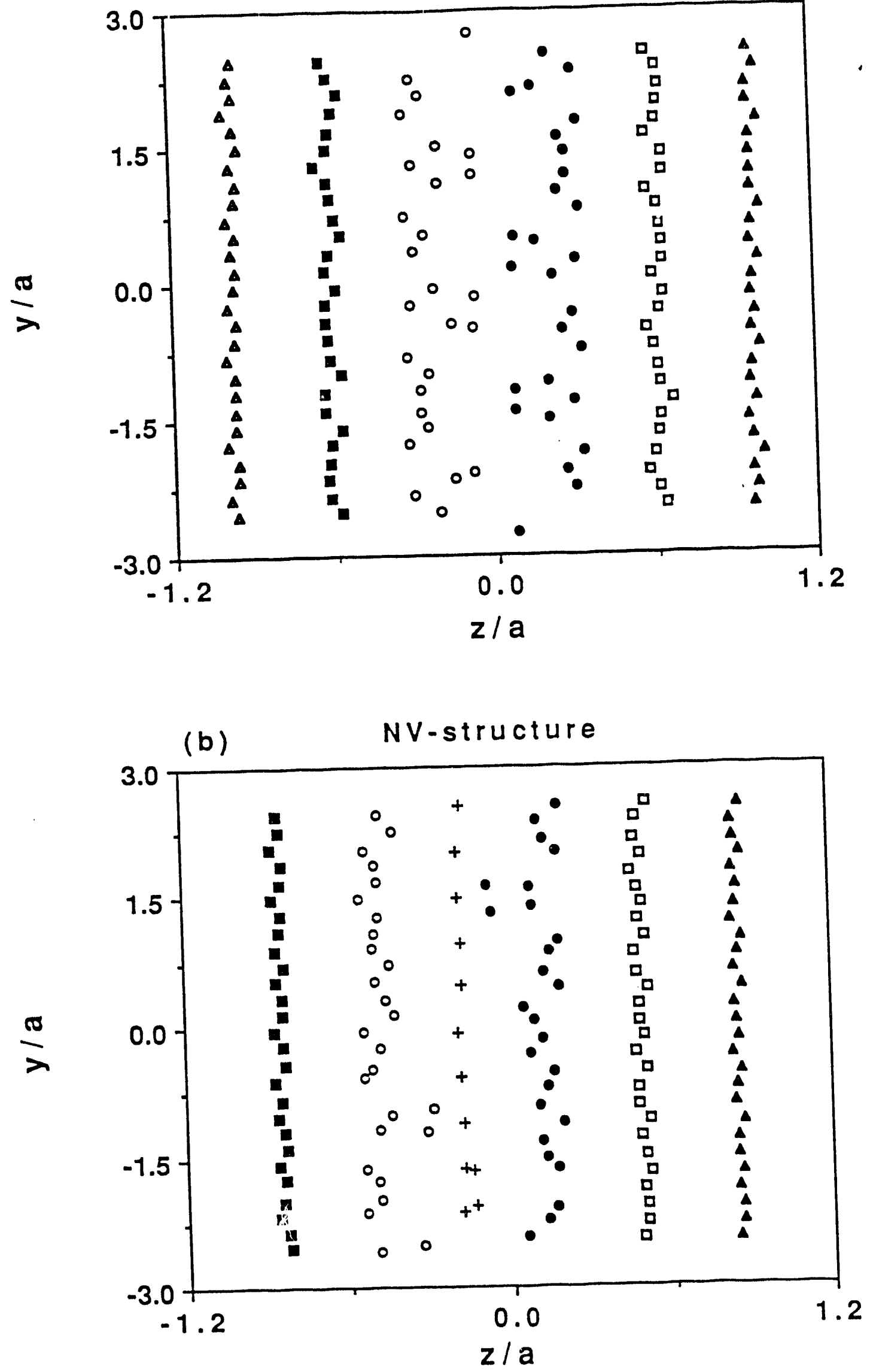

$F_{y .3}$ 

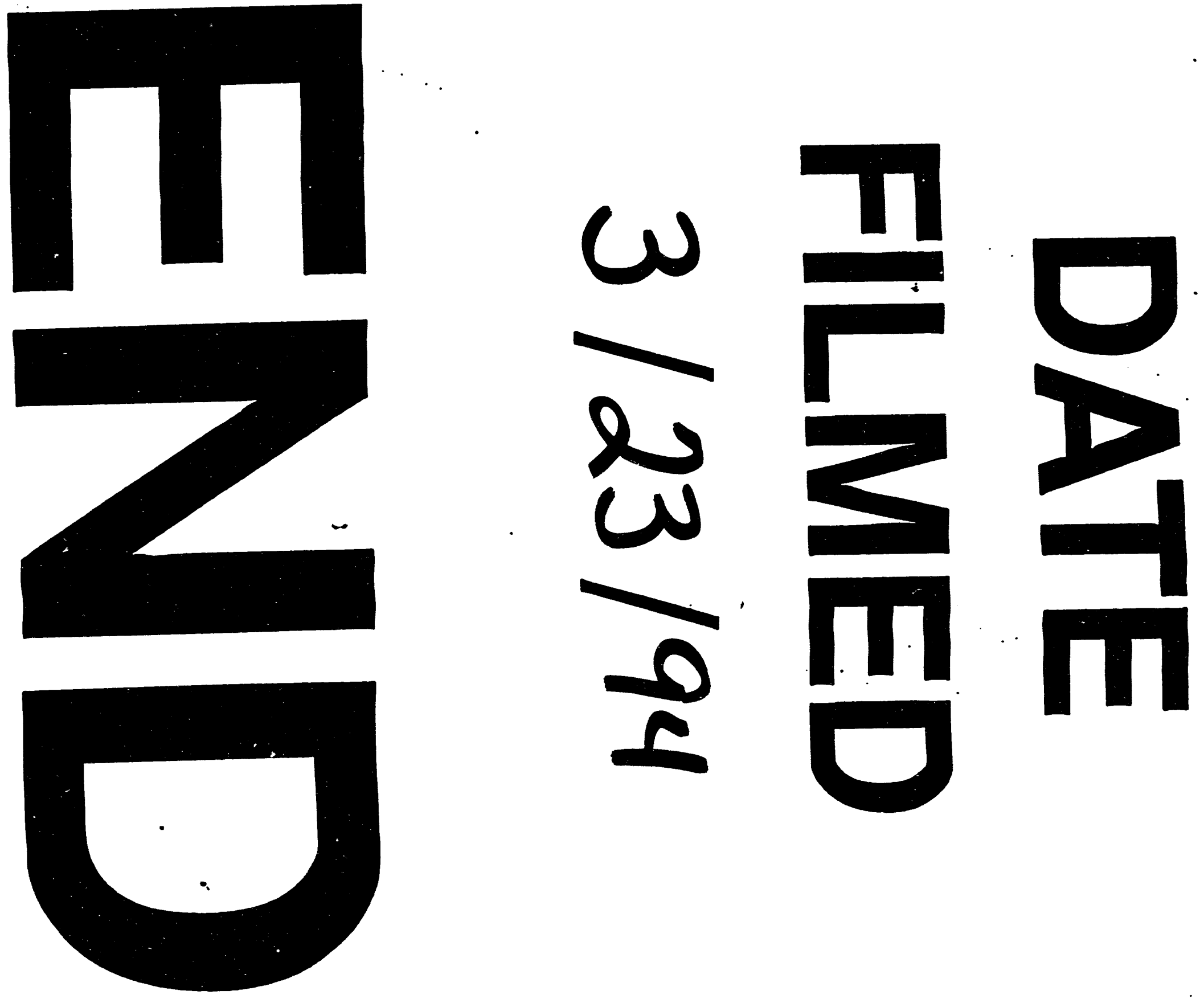
